\title{
Tuberculosis-specific CD8 cells in HLA A*02-positive TB- and LTBI patients
}

\author{
Andreas Flø $\mathrm{e}^{1}$, Liselotte Brix ${ }^{2}$, Christian Wejse ${ }^{3}$, Ole Hilberg ${ }^{1}$ \\ 1: Department of Respiratory Medicine and Allergy, Aarhus University Hospital, Aarhus, Denmark \\ 2: Immudex ApS, Copenhagen, Denmark \\ 3: Department of Infectious Diseases, Aarhus University Hospital, Skejby, Denmark and GloHAU Center for Global Health, Aarhus University, \\ Denmark
}

Background: Understanding the CD8+ response against Mycobacterium tuberculosis (MTB) may be a key to improved TB diagnostics and vaccine development.

Aims and Objectives: To detect a CD8+ T-cell response against Mycobacterium tuberculosis (MTB) in active tuberculosis (TB) and latent TB (LTBI), in HLA A*02 positive patients.

Methods: We identified possible epitopes (antigen fragments) from 9 MTB antigens (Ag85B, ESAT-6, EsxH, Hsp65, EsxJ, rv1490, rv1614, rv2626c and $16 \mathrm{kDa}$ antigen) by searching the literature, and by computer prediction of likely binding to MHC-1. We selected 24 epitope candidates, from which we constructed MHC multimers (Dextramers). Peripheral blood mononuclear cells (PBMC) from 7 TB-patients, 16 LTBI patients and 8 MTB-exposed, IGRA-negative, healthy subjects (HE), all HLA A*02 positive, were stained with the Dextramers and with anti-CD8 and anti-CD3, and analyzed on a flow cytometer. The MTB epitopes were analyzed in 5 pools (3-7 epitopes each). Positive responses included $>0.001 \%$ of CD8+, CD3+ cells, supported by inspection of flow cytometry plots.

Results: MTB-specific CD8+ T-cells were detected more often in TB patients (57\%) than in LTBI patients (41\%) and in HE (25\%), though differences were insignificant. CD8+ responses were seen against a broad spectrum of antigens, including not previously described Rv2626c epitopes. Reactivity against multiple antigens was observed in 3 of 7 LTBI patients and in 2 of 4 TB patients with detectable CD8+ responses.

Conclusion: Broad diversity in epitope-specificity was seen among MTB-specific CD8+ T-cells in HLA A*02, consistent with previous reports. A trend of more common detection of MTB-specific CD8+ cells in TB than in LTBI and HE was found. 